\title{
Control of the metal/gas ion ratio incident at the substrate plane during high-power impulse magnetron sputtering of transition metals in Ar
}

Grzegorz Greczynski, Igor Zhirkov, Ivan Petrov, J oseph E Greene and J ohanna Rosén

The self-archived postprint version of this journal article is available at Linköping University Institutional Repository (DiVA):

http:/ / urn.kb.se/ resolve?urn=urn:nbn:se:liu:diva- 142963

N.B.: When citing this work, cite the original publication.

Greczynski, G., Zhirkov, I., Petrov, I., Greene, J. E, Rosén, J., (2017), Control of the metal/ gas ion ratio incident at the substrate plane during high-power impulse magnetron sputtering of transition metals in Ar, Thin Solid Films, 642, 36-40. https:// doi.org/ 10.1016/j.tsf.2017.09.027

Original publication available at:

https:// doi.org/ 10.1016/j.tsf.2017.09.027

Copyright: Elsevier

http:// www.elsevier.com/

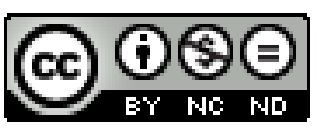




\title{
Control of the metal/gas ion ratio incident at the substrate plane during high-power impulse magnetron sputtering of transition metals in Ar
}

\author{
G. Greczynski, ${ }^{1}$ I. Zhirkov, ${ }^{1}$ I. Petrov, ${ }^{1,2}$ J.E. Greene, ${ }^{1,2,3}$ and J. Rosen ${ }^{1}$ \\ ${ }^{1}$ Department of Physics (IFM), Linköping University, SE-581 83 Linköping, Sweden \\ ${ }^{2}$ Materials Science Department and Frederick Seitz Materials Research Laboratory, University of \\ Illinois, Urbana, Illinois 61801 \\ ${ }^{3}$ Department of Physics, University of Illinois, Urbana, Illinois 61801, USA
}

\begin{abstract}
High-power impulse magnetron sputtering (HiPIMS) of materials systems with metal/gasatom mass ratios $m_{M e} / m_{g}$ near, or less than, unity presents a challenge for precise timing of synchronous substrate-bias pulses to select metal-ion irradiation of the film and, thus, reduce stress while increasing layer density during low-temperature growth. The problem stems from high gasion fluxes $F_{g^{+}}(t)$ at the substrate, which overlap with metal-ion fluxes $F_{M e^{+}}(t)$. We use energyand time-dependent mass spectrometry to analyze $F_{M e^{+}}(t)$ and $F_{g^{+}}(t)$ for Group IVb transitionmetal targets in Ar and show that the time-and energy-integrated metal/gas ion ratio $\mathrm{N}_{\mathrm{Me}^{+}} / \mathrm{N}_{\mathrm{Ar}^{+}}$ at the substrate can be controlled over a wide range by adjusting the HiPIMS pulse length $\tau_{O N}$, while maintaining the peak target current density $J_{T \text {,peak }}$ constant. The effect is a consequence of severe gas rarefaction which scales with $J_{T}(t)$. For Ti-HiPIMS, terminating the discharge at the maximum $J_{T}(t)$, corresponding to $\tau_{O N}=30 \mu \mathrm{s}$, there is an essentially complete loss of $\mathrm{Ar}^{+}$ion intensity, yielding $N_{T i^{+}} / N_{A r^{+}} \sim 60$. With increasing $\tau_{O N}, J_{T}(t)$ decreases and $N_{T i^{+}} / N_{A r^{+}}$gradually decays, due to Ar refill, to $\sim 1$ with $\tau_{O N}=120 \mu$ s. Time-resolved ion-energy distribution functions confirm that the degree of rarefaction depends on $\tau_{\mathrm{ON}}$ : for shorter pulses, $\tau_{\mathrm{ON}}<60 \mu$ s, the original sputtered-atom Sigmund-Thompson energy distributions are preserved long after the HiPIMS pulse, which is in distinct contrast to longer pulses, $\tau_{O N} \geq 60 \mu \mathrm{s}$, for which the energy distributions collapse into narrow thermalized peaks. Thus, optimizing the HiPIMS pulse width minimizes the
\end{abstract}


gas-ion flux to the substrate independent of $m_{M e} / m_{g}$.

corresponding author: grzgr@ifm.liu.se; phone: +46 13281213

Keywords: HiPIMS; HPPMS; PVD; sputtering; gas rarefaction; transition metals 


\section{Introduction}

During the past decade, High Power Impulse Magnetron Sputtering (HiPIMS) [1] has been the subject of extensive investigations $[2,3,4,5,6]$ due to an intrinsic ability to deliver large fluxes of metal ions. Moreover, the strong gas rarefaction $[7,8,9,10,11]$ immediately in front of the target during HiPIMS discharges provides inherent time and energy separation of gas- and metal-ion fluxes incident at the substrate $[12,13]$. This yields the potential to provide new film growth pathways, and additional control over film composition and physical properties, beyond that available with magnetically-unbalanced magnetron sputtering $[14,15]$, by synchronizing the substrate bias to the metal-ion portions of HiPIMS pulses $[16,17,18]$. Metal-ions, as opposed to noble-gas ions, are primarily incorporated at film lattice sites which, together with dramaticallyreduced concentrations of trapped gas ions, results in lower compressive stresses in as-deposited layers [16,19]. Moreover, the metal-ion mass, incident flux, and impact energy can be independently controlled to tune momentum transfer and provide the recoil density and energy necessary to eliminate film porosity at low deposition temperatures [20,21]. Optimizing the results in experiments such as those referenced above, primarily carried out during reactive transitionmetal (TM) nitride film growth, requires a detailed knowledge of the time evolution of metal- and gas-ion fluxes incident at the substrate plane in order to precisely tune the synchronous bias pulse [22].

Recently, we analyzed the evolution of metal- and gas-ion fluxes incident at the substrate during HiPIMS sputtering of Groups IVb and VIb TM targets in Ar [23]. We found that the timeand energy-integrated metal/gas ion ratio $N_{M e^{+}} / N_{A r^{+}}$increased with increasing peak target current density, due to gas rarefaction, and exhibited a strong dependence on metal/gas-atom mass ratio $m_{M e} / m_{g} ; N_{M e^{+}} / N_{A r^{+}}$ranged from $\sim 1$ for Ti $\left(m_{T i} / m_{A r}=1.20\right)$ to $\sim 100$ for $\mathrm{W}\left(m_{W} / m_{A r}=4.60\right)$. 
This difference in gas density was also reflected in the time evolution of the original sputteredmetal Sigmund-Thompson ion energy distribution functions (IEDFs), which only collapsed into a narrow thermalized peak for lighter-mass TMs, and remained unchanged for heavier sputtered species with $m_{M e} / m_{g} \gg 1$. Thus, the use of higher-mass (relative to the metal) noble-gases renders metal/gas combinations with $m_{M e} / m_{g}$ near, or less than, unity a challenge for precise timing of synchronous substrate-bias pulses, in order to reduce film stress while increasing densification.

Here, we present the results of an investigation which provides a strategy to remedy the issue outlined above. We show, for Group IVb TM targets sputtered in Ar, that by adjusting the duration of the HiPIMS pulse $\tau_{O N}$, while maintaining the peak target current density $J_{T, p e a k}$ constant, the time- and energy-integrated metal/gas ion ratio $N_{M e^{+}} / N_{A r^{+}}$incident at the substrate plane can be controlled over a wide range, as a consequence of severe gas rarefaction which scales with the time-dependent target current density $J_{T}(t)$. The effect is particularly important for power-supply-limited discharges characterized by peak-shaped current pulses. We show, and explain why, it is highly beneficial to terminate the HiPIMS pulse at the maximum $J_{T}(t)$ value, which minimizes the gas-ion flux to the substrate and allows for much easier control of metal-ion fluxes even for challenging metal/gas combinations with $m_{M e} / m_{g} \lesssim 1$.

\section{Experimental Details}

Time-dependent in-situ mass- and energy-spectroscopy analyses of ion fluxes during HiPIMS sputtering of Ti, Zr, and Hf targets (99.99 \% pure) in Ar are performed using a Hiden Analytical EQP1000 instrument. Experiments are carried out in a CemeCon CC800/9 magnetron sputtering system, equipped with integrated HiPIMS power supplies, modified to allow mass spectrometry 
analyses at the same target/substrate separation used during film growth. One rectangular $8.8 \times 50$ $\mathrm{cm}^{2}$ target is moved from its original position to the center of the sputtering chamber, while the opposite cathode is replaced with a mass spectrometer probe. The probe orifice points toward the center of the target $18 \mathrm{~cm}$ away. The spectrometer axis is perpendicular to the target. The system base pressure is $0.2 \mathrm{mPa}\left(1.5 \times 10^{-6}\right.$ Torr $)$, and the Ar sputtering pressure is maintained constant at $P=0.4 \mathrm{~Pa}$ (3 mTorr). The HiPIMS pulse lengths investigated are 20, 30, 40, 60, 80, 100, and 120

$\mu$ s at a frequency of $300 \mathrm{~Hz}$. This corresponds to the average HiPIMS power ranging from 0.8 to $3 \mathrm{~kW}$ for Ti, 0.6 to $3.5 \mathrm{~kW}$ for $\mathrm{Zr}$, and from 1.2 to $3.8 \mathrm{~kW}$ for the Hf target. Ion-energy distribution functions $I_{\mathrm{Me}^{n+}}\left(E_{i}\right)$ are recorded in HiPIMS mode for $\mathrm{Me}^{n+}(n=1,2, \ldots)$ metal and gas ions during 100 consecutive pulses such that the total acquisition time per data point is $1 \mathrm{~ms}$. The ion energy is scanned in $0.5 \mathrm{eV}$ steps from $E_{i}=1$ to $50 \mathrm{eV}$. Additional details regarding the IEDF measurements, including ion time-of-flight corrections, are given in Refs. 13,24. Target current and voltage waveforms during film growth are acquired with a Tektronix $500 \mathrm{MHz}$ bandwidth digital oscilloscope.

\section{Results and Discussion}

Figure 1 shows target voltage and current density waveforms recorded during Ti HiPIMS as a function of pulse durations $\tau_{\text {ON }}$ from 20 to $120 \mu$ s. The voltage oscillations that occur upon pulse shutdown, as well as related low-amplitude current transients, are artefacts due to the system modification required in order to perform mass spectrometry analyses (see Experimental Details). For all $\tau_{\mathrm{ON}}$ values, the target voltage $V_{T}(t)$ is $650 \mathrm{~V}$ at $t=0$ and decreases with time, due to the size of the capacitor bank with respect to the target area, to reach $550,465,390$, and $300 \mathrm{~V}$ with $\tau_{\mathrm{ON}}=$ $20,30,40$, and $60 \mu$ s, respectively, or to saturate at $260 \mathrm{~V}$ for all pulses with $\tau_{O N} \geq 80 \mu$ s. Following 
plasma ignition at $t=3 \mu \mathrm{s}$, the target current density $J_{T}(t)$, Fig. 1(b), increases rapidly to a

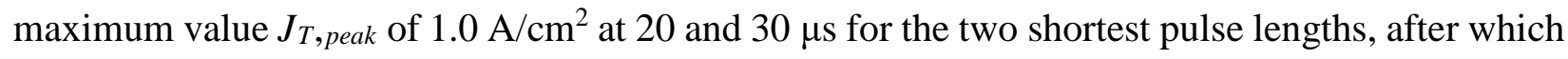
it rapidly decays to zero within $\sim 2 \mu$ s as the target voltage is switched off. For $\tau_{O N}>30 \mu$ s, $J_{T}(t)$ gradually decreases after reaching $J_{T \text {,peak }}$ at $t=30 \mu \mathrm{s}$, to $0.8,0.3$, and $0.06 \mathrm{~A} / \mathrm{cm}^{2}$ with $\tau_{O N}=40$, 60 , and $80 \mu \mathrm{s}$; then, discharge is terminated. In the case of even longer pulses, $\tau_{\mathrm{ON}}>80 \mu \mathrm{s}, J_{T}(t)$ decays to essentially zero at the end of the HiPIMS pulse, as $V_{T}(t)$ becomes too low to sustain the discharge.

Time-dependent intensities of the primary-ion fluxes incident at the substrate plane during Ti-HiPIMS $\left(\mathrm{Ti}^{+}, \mathrm{Ti}^{2+}\right.$, and $\left.\mathrm{Ar}^{+}\right)$are plotted in Fig. 2 with a $10 \mu$ s resolution for HiPIMS pulse durations $\tau_{O N}$ of 30 and $120 \mu$ s. While the former corresponds to terminating the pulse at the maximum current, the latter represents the case in which the discharge self-terminates, and is characteristic of all pulses with $\tau_{\mathrm{ON}}>80 \mu \mathrm{s}$. In both cases, 30 and $120 \mu \mathrm{s}$, the peak target current density is $1.0 \mathrm{~A} / \mathrm{cm}^{2}$. Zero on the time axis corresponds to the onset of the cathode voltage pulse, while each data point at time $t$ represents the number of ions collected during the interval from $t-5$ to $t+5 \mu \mathrm{s}$. With $\tau_{O N}=120 \mu$ s (Fig. 2, bottom panel), the metal-ion flux $F_{T i^{+}}(t)$ is composed of a relatively narrow peak which dominates the total ion flux to the substrate from $t=30$ to $90 \mu \mathrm{s}$, and a lower-intensity tail extending to $\sim 260 \mu$ s due to thermalized $\mathrm{Ti}^{+}$ions. The gas-ion flux $F_{A r^{+}}(t)$ exhibits a time evolution consistent with previous reports [12,16], reaching a maximum during the early stages of the pulse, 0-40 $\mu$ s, indicating that the plasma is initially maintained primarily by gas ions. After a rapid decay of the metal-ion flux, $F_{A r^{+}}(t)$ again dominates the ion flux from $t=100$ to $200 \mu \mathrm{s}$ as the Ar density increases in front of the sputtering target due to a rapid decrease in $J_{T, p e a k}$ 
With $\tau_{O N}=30 \mu$ s (Fig. 2, top panel), the striking difference is an essentially complete loss of $\mathrm{Ar}^{+}$ion intensity. The energy and time-integrated metal/gas ion ratio $N_{\mathrm{Ti}^{+}} / N_{\mathrm{Ar}^{+}}$, defined as $\int F_{T i^{+}}(t) d t / \int F_{A r^{+}}(t) d t$ with the integral extending from $t=0$ to $300 \mu$ s, increases from 1 with $\tau_{\mathrm{ON}}=120 \mu$ s to 60 with $\tau_{\mathrm{ON}}=30 \mu \mathrm{s}$. In addition, $F_{T i^{+}}(t)$ at $\tau_{\mathrm{ON}}=30 \mu$ s exhibits zero thermalization intensity at $t>120 \mu \mathrm{s}$, dramatically different than observed with $\tau_{\mathrm{ON}}=120 \mu \mathrm{s}$ pulses. The width of the $F_{T i^{+}}(t)$ distribution at $10 \%$ intensity, which includes the entire time period over which metal ions are incident at the substrate, is reduced from $~ 100$ to $70 \mu$ s upon shortening the HiPIMS pulse from 120 to $30 \mu$ s. Clearly, decreasing the HiPIMS pulse length results in much stronger gas rarefaction. Termination of the discharge once $J_{T}(t)$ reaches a maximum prevents the rapid Ar refill which occurs during longer pulses characterized by a gradual $J_{T}$ decay (see Fig. 1(b)). Interestingly, the initial $F_{A r^{+}}(t)$ peak at $t=0-40 \mu$ s also disappears with $\tau_{O N}=30 \mu \mathrm{s}$, which indicates that this feature is strongly affected by the residual $\mathrm{Ar}^{+}$density from the preceding pulse.

Fig. 3 presents $\mathrm{Ti}^{+}$IEDFs acquired at the substrate plane over consecutive $20-\mu$ s time intervals during and after (a) $30-\mu$ s and (b) $120-\mu$ s HiPIMS pulses while sputtering in Ar. Since metal IEDFs depend upon the efficiency of mass transport through the gas phase, analyses of $I_{T i^{+}}\left(E_{i}\right)$ time evolutions reveal essential differences among short and long HiPIMS pulses. For $\tau_{O N}$ $=120 \mu \mathrm{s}$, Ti metal-ion IEDFs in the early stages $(t<100 \mu \mathrm{s})$, resemble very broad SigmundThompson sputtered-species energy distributions [25,26]; that is, $I_{T i^{+}}\left(E_{i}\right) \propto E_{i} /\left(E_{i}+E_{b}\right)^{3}$. (in which $E_{b}$ denotes the surface-atom binding energy) [25]. The original energy distribution of sputter-ejected atoms is preserved since these species undergo few or no collisions due to plasma rarefaction [27]. During the later phases of $120 \mu$ s pulses $(t>100 \mu \mathrm{s}), I_{T i^{+}}\left(E_{i}\right)$ IEDFs gradually collapse to narrow, low-energy peaks at 2-3 eV (reflecting the potential difference between the bulk plasma potential and the grounded orifice), as a result of thermalization, in which the sputtered 
species lose energy via collisions with noble-gas atoms, as the target current density and, hence, the degree of rarefaction decreases (i.e., the local rare-gas pressure increases).

In contrast, for shorter HiPIMS pulses, $\tau_{O N}=30 \mu$ s, terminated at the $J_{T}(t)$ peak maximum, IEDFs in Fig. 3(a) reveal that the original broad Sigmund-Thompson energy distributions are preserved throughout the entire measurement period up to $300 \mu$ s, and beyond. $I_{T i^{+}}\left(E_{i}\right)$ IEDFs do not collapse into a narrow thermalized peak, as observed with longer HiPIMS pulses. Instead, a gradual loss in intensity occurs and even the ions arriving at $t>160 \mu$ s, thus $130 \mu$ s after the HiPIMS pulse, have an average energy of $12 \mathrm{eV}$. The dramatic decrease in thermalized ion density, despite the fact that the thermalization distance $\lambda_{\text {th }}$ at $P=0.4 \mathrm{~Pa}$ (3 mTorr) is significantly shorter than the target-orifice distance $[23,28]$, indicates severe rarefaction and is consistent with the fact that the energy and time-integrated metal/gas ion ratio $N_{\mathrm{Ti}^{+}} / N_{\mathrm{Ar}^{+}}$increases with shortening of the HiPIMS pulse, as discussed above.

$N_{\mathrm{Me}^{+}} / \mathrm{N}_{\mathrm{Ar}^{+}}$ratios are plotted in Fig. 4 as a function of the HiPIMS pulse length for all Group IVb TMs sputtered in Ar with a constant peak target current density $J_{T, p e a k}=1.0 \mathrm{~A} / \mathrm{cm}^{2}$. Clearly, the trends outlined above for $\mathrm{Ti}$ also hold for $\mathrm{Zr}$ and $\mathrm{Hf}$. At the longest pulse length investigated, $120 \mu \mathrm{s}, N_{\mathrm{Me}^{+}} / N_{A r^{+}}$increases with increasing metal/gas-atom mass ratio $m_{M e} / m_{g}$ as a result of decreasing gas density in front of the sputtering target (increased rarefaction). This is due to the fact that the metal/gas collision cross-section increases with increasing sputtered-atom mass, resulting in shorter mean free paths [23], increased momentum transfer, and hence more effective gas heating. As the HiPIMS pulse-length decreases, $N_{\mathrm{Me}^{+}} / N_{\mathrm{Ar}}+$ exhibits an increase for all TMs, and reaches a maximum with $\tau_{O N} \simeq 30-40 \mu$ s, corresponding to maxima in $J_{T}(t)$. The relative increase in the total metal/gas ion ratio decreases with increasing $m_{M e} / m_{g}$, from $60 \times$ for Ti, to a 
factor of $3 \times$ and $1.6 \times$ for $\mathrm{Zr}$ and Hf. This is a consequence of the fact that the sputtering gas is severely diluted for heavier metal species, even at longer HiPIMS pulses.

Figure 5 shows time-dependent intensities of metal-ion fluxes incident at the substrate plane during and after 30- $\mu$ s HiPIMS pulses while sputtering Group IVb TM targets, each at a current density of $1 \mathrm{~A} / \mathrm{cm}^{2}$. An important observation for metal-ion-synchronized HiPIMS is that the time delays between the end of the target current pulses and subsequent $F_{M e^{+}}(t)$ peaks increase with increasing metal-ion mass; from $25 \mu$ s for $\mathrm{Ti}$, to $29 \mu$ s for $\mathrm{Zr}$, and $60 \mu$ s for Hf. Consequently, metal-ion peaks are broader for heavier ions. The width of the $F_{M e^{+}}(t)$ distribution at $10 \%$ intensity, which includes the entire time period over which metal ions are incident at the substrate, varies from $\sim 70 \mu$ s for $\mathrm{Ti}$, to $\sim 107 \mu$ s for $\mathrm{Zr}$, and $\sim 154 \mu$ s for Hf. Both effects stem from the fact that the times-of-flight from the target increase with increasing $m_{M e}$.

The above results have direct implications for HiPIMS film growth employing synchronous biasing. Previously, we established for $120-\mu$ s HiPIMS pulses that the precise tuning of bias pulse length and offset, used to control the energy and momentum of metal ions incident at the growing film surface, is much more critical for metal/gas combinations with $m_{M e} / m_{g} \lesssim 1$, while with $m_{M e} / m_{g} \gg 1$, the length of the synchronous bias pulse is controlled by the metal-ion time of flight [23]. Thus, for heavier species, there is more flexibility in choosing pulse shapes in order to maximize the metal-ion flux while minimizing gas-ion irradiation incident at the substrate, since the width of the bias pulse can be increased with increasing ion mass for a given target/substrate separation. In this respect, metal/gas combinations with $m_{M e} / m_{g} \lesssim 1$ present significant challenges. For such cases, the choice of pulse offset and length in order to obtain the best time separation between metal- and gas-ion fluxes at the substrate plane is critical and depends on the HiPIMS peak target current. 
The results presented here provide a solution. Tuning the HiPIMS pulse length yields control of $N_{M e^{+}} / N_{A r^{+}}$over a wide range, which is particularly useful for metal/gas combinations with low $m_{M e} / m_{g}$ ratios such as Ti/Ar. With the pulse length adjusted to terminate the current pulse at $t$ corresponding to the maximum in $J_{T}(t)$, gas rarefaction effects are maximized which, in turn, provides $N_{M e^{+}} / N_{A r^{+}}$ratios characteristic of metal/gas-atom combinations with $m_{M e} / m_{g} \gg 1$. Hence, shorter HiPIMS pulses offer more flexibility in precise timing of synchronous substratebias pulses, such that the width of the synchronous bias pulse is essentially controlled by the metalion time of flight, independent of the metal/gas-atom mass ratio.

\section{Conclusions}

In summary, we have used energy- and time-dependent mass spectrometry analyses to determine metal- and gas-ion fluxes incident at the substrate plane during synchronized-bias HiPIMS sputtering of Group IVb transition-metal targets in Ar as a function of pulse width. We demonstrated that the time-and energy-integrated metal/gas ion ratio $N_{M e^{+}} / N_{A r^{+}}$can be controlled over wide range by adjusting the HiPIMS pulse width $\tau_{O N}$ while maintaining the peak target current density constant. This additional tunability in controlling ion fluxes incident at the substrate is due to enhanced gas rarefaction which scales with the target current density $J_{T}(t)$. For HiPIMS with current pulses for which $J_{T}(t)$ exhibits a gradual decay after reaching a peak value $J_{T, p e a k}$, due to the size of the capacitor bank with respect to the target area, gas density is gradually restored. Thus, terminating the discharge at $J_{T \text {,peak }}$ results in maximizing the $\mathrm{N}_{\mathrm{Me}^{+}} / N_{\mathrm{Ar}}+$ ratio at the substrate. This is particularly useful for metal/gas combinations with low $m_{M e} / m_{g}$ ratios such as $\mathrm{Ti} / \mathrm{Ar}\left(m_{M e} / m_{g}\right.$ $=1.2)$ for which $N_{T i^{+}} / N_{A r^{+}}$varies from $\sim 1$ with $\tau_{O N}=120 \mu$ s to $\sim 60$ for $\tau_{O N}=30 \mu$ s. These results are important for controlling metal-ion-synchronized HiPIMS film growth, where the use of 
synchronized substrate-bias pulses, in order to reduce the trapped-Ar concentration in the growing film, requires that the bias pulse length and the offset be precisely timed. We demonstrated that by optimizing the HiPIMS pulse length, the ion flux to the substrate is dominated by metal ions, hence there is more flexibility in choosing bias pulse shapes. The length of the synchronous bias pulse is controlled by the metal-ion time of flight, independent of the choice of metal/gas combinations.

We expect that the strategy presented in this paper should have a pronounced impact on film properties, as it allows for independent control of metal-ion energy and momentum, which can be decoupled from that of gas ions, even for short pulses. Since varying the HiPIMS pulse length has a large impact on the metal/gas ion ratio for each target studied (Ti, $\mathrm{Zr}$, and $\mathrm{Hf}$ ), we believe the effect is general, and should occur for all industry-relevant material systems. In reactive processes, metal reactivity, which controls the rate of compound formation at the target, is expected to be a decisive parameter, as it directly affects the target sputtering rate and, hence, the extent of gas rarefaction. Thus, the effect of pulse length on the metal/gas ion ratio in reactive mode is expected to be less pronounced for materials that are highly reactive.

\section{Acknowledgements}

Financial support from the Swedish Research Council VR Grant 2014-5790, an Åforsk foundation grant \#16-359, the Swedish Government Strategic Research Area in Materials Science on Functional Materials at Linköping University (Faculty Grant SFO Mat LiU No. 2009 00971), the Knut and Alice Wallenberg foundation Fellowship Grant and Project funding (KAW 2015.0043), and Carl Tryggers Stiftelse contracts CTS 15:219 and CTS 14:431 is gratefully acknowledged. 


\section{Figure captions}

Fig. 1 (Figure in color online) (a) Target voltage $V_{T}(t)$, and (b) target current density $J_{T}(t)$ waveforms, recorded during HiPIMS sputtering of a Ti target in Ar at $0.4 \mathrm{~Pa}$ (3 mTorr), as a function of the target pulse width $\tau_{O N}$ varied from $20 \mu$ s to $120 \mu$ s.

Fig. 2. (Figure in color online) Time evolution of the energy-integrated $\mathrm{Ti}^{+}, \mathrm{Ti}^{2+}$, and $\mathrm{Ar}^{+}$ion fluxes $F(t)$ incident at the substrate plane during and after HiPIMS pulses while sputtering a $\mathrm{Ti}$ target in Ar at $0.4 \mathrm{~Pa}$ (3 mTorr) with (a) $30 \mu$ s and (b) $120 \mu$ s HiPIMS pulses. The peak target current density $J_{T, p e a k}$ is maintained constant at $1.0 \mathrm{~A} / \mathrm{cm}^{2}$. Target current pulses $J_{T}(t)$, shown in grey (dashed line), are scaled to match the $F_{T i^{+}}(t)$ intensity in order to facilitate comparison.

Fig. 3. (Figure in color online) $\mathrm{Ti}^{+}$ion energy distribution functions (IEDFs) recorded at the substrate position during HiPIMS sputtering of Ti target in Ar at 0.4 Pa (3 mTorr). The IEDFs are acquired during 20- $\mu$ s time intervals over the time period $t$ from 0 (pulse ignition) to $200 \mu$ s. The HiPIMS pulse length is (a) $30 \mu$ s and (b) $120 \mu$ s.

Fig. 4. (Figure in color online) Time- and energy-integrated metal/gas ion ratios $N_{\mathrm{Me}^{+}} / N_{\mathrm{Ar}^{+}}$ incident at the substrate plane as a function of pulse length $\tau_{O N}$ during HiPIMS sputtering of $\mathrm{Ti}, \mathrm{Zr}$, and $\mathrm{Hf}$ targets in Ar at $0.4 \mathrm{~Pa}$ (3 mTorr). 
Fig. 5. (Figure in color online) Time evolution of the energy-integrated Group IVb Ti, Zr, and Hf metal-ion fluxes $F_{\mathrm{Me}^{+}}(t)$ incident at the substrate plane during and after $30 \mu$ s HiPIMS pulses while sputtering elemental targets in Ar at $0.4 \mathrm{~Pa}$ (3 mTorr) with a peak target current density $J_{T, p e a k}=1.0 \mathrm{~A} / \mathrm{cm}^{2}$. 


\section{References}

[1] V. Kouznetsov, K. Macak, J. M. Schneider, U. Helmersson and I. Petrov, A novel pulsed magnetron sputter technique utilizing very high target power densities, Surf. Coat. Technol. 122 (1999) 290-293

[2] S. Loquai, O. Zabeida, J. E. Klemberg-Sapieha, and L. Martinu, Flash post-discharge emission in a reactive HiPIMS process, Appl. Phys. Lett. 109 (2016) 114101

[3] P. Raman, J. Weberski, M. Cheng, I. Shchelkanov, and D. N. Ruzic, A high power impulse magnetron sputtering model to explain high deposition rate magnetic field configurations, J. Appl. Phys. 120 (2016) 163301

[4] R. Hippler, Z. Hubicka, M. Cada, P. Ksirova, H. Wulff, C. A. Helm, and V. Stranak, Angular dependence of plasma parameters and film properties during high power impulse magnetron sputtering for deposition of $\mathrm{Ti}$ and $\mathrm{TiO}_{2}$ layers, J. Appl. Phys. 121 (2017) 171906

[5] A. Anders, Tutorial: Reactive high power impulse magnetron sputtering (R-HiPIMS), J. Appl. Phys. 121 (2017) 171101

[6] A. Hecimovic, N. Britun, S. Konstantinidis, and R. Snyders, Sputtering process in the presence of plasma selforganization, Appl. Phys. Lett. 110 (2017) 014103

[7] K. Sarakinos, J. Alami, S. Konstantinidis, High power pulsed magnetron sputtering: A review on scientific and engineering state of the art, Surf. Coat. Technol. 204 (2010) 1661-1684

[8] U. Helmersson, M. Lattemann, J. Bohlmark, A. P. Ehiasarian, and J. T. Gudmundsson, Ionized physical vapor deposition (IPVD): A review of technology and applications, Thin Solid Films 513 (2006) 1-24

[9] D. Horwat, A. Anders, Compression and strong rarefaction in high power impulse magnetron sputtering discharges, J. Appl. Phys. 108 (2010) 123306

[10] A. Anders, Discharge physics of high power impulse magnetron sputtering, Surf. Coat. Technol. 205 (2011) S1S9

[11] N. Britun, S. Konstantinidis, R. Snyders, An Overview on Time-Resolved Optical Analysis of HiPIMS Discharge, Plasma Processes and Polymers 12 (2015) 1010-1025

[12] K. Macak, V. Kouznetsov, J. Schneider, U. Helmersson, I. Petrov, Ionized sputter deposition using an extremely high plasma density pulsed magnetron discharge, J. Vac. Sci. Technol. A 18 (2000) 1533

[13] G. Greczynski and L. Hultman, Time and energy resolved ion mass spectroscopy studies of the ion flux during high power pulsed magnetron sputtering of $\mathrm{Cr}$ in $\mathrm{Ar}$ and $\mathrm{Ar} / \mathrm{N}_{2}$ atmospheres, Vacuum 84 (2010) 1159-1170

[14] I. Petrov, L. Hultman, J.-E. Sundgren, and J. E. Greene, Polycrystalline TiN films deposited by reactive bias magnetron sputtering: Effects of ion bombardment on resputtering rates, film composition, and microstructure, J.

Vac. Sci. Technol. A 10 (1992) 265

[15] I. Petrov, P.B. Barna, L. Hultman, J.E. Greene, Microstructural evolution during film growth, J. Vac. Sci.

Technol. A 21 (2003) 117

[16] G. Greczynski, J. Lu, J. Jensen, I. Petrov, J.E. Greene, S. Bolz, W. Kölker, Ch. Schiffers, O. Lemmer and L. Hultman, J. Vac. Sci. Technol. A 30 (2012) 061504

[17] G. Greczynski, J. Lu, J. Jensen, S. Bolz, W. Kölker, Ch. Schiffers, O. Lemmer, J.E. Greene, and L. Hultman, A review of metal-ion-flux-controlled growth of metastable TiAIN by HIPIMS/DCMS co-sputtering, Surf. Coat.

Technol. 257 (2014) 15-25

[18] G. Greczynski, J. Patscheider, J. Lu, B. Alling, A. Ektarawong, J. Jensen, I. Petrov, J. E. Greene, L. Hultman, Control of $\mathrm{Ti}_{1-x} \mathrm{Si}_{x} \mathrm{~N}$ nanostructure via tunable metal-ion momentum transfer during HIPIMS/DCMS co-deposition,

Surf. Coat. Technol. 280 (2015) 174-184

[19] G. Greczynski, J. Lu, J. Jensen, I. Petrov, J.E. Greene, S. Bolz, W. Kölker, Ch. Schiffers, O. Lemmer and L. Hultman, Strain-free, single-phase metastable Ti0.38Al0.62N alloys with high hardness: metal-ion energy vs. momentum effects during film growth by hybrid high-power pulsed/dc magnetron cosputtering, Thin Solid Films 556 (2014) 87-98

[20] G. Greczynski, J. Lu, S. Bolz, W. Kölker, Ch. Schiffers, O. Lemmer, I. Petrov, J.E. Greene, L. Hultman, Novel strategy for low-temperature, high-rate growth of dense, hard, and stress-free refractory ceramic thin films, J. Vac. Sci. Technol. A 32 (2014) 041515

[21] H. Fager, O. Tengstrand, J. Lu, S. Bolz, B. Mesic, W. Kölker, Ch. Schiffers, O. Lemmer, J. E. Greene, L. Hultman, I. Petrov, G. Greczynski, Low-temperature growth of dense and hard $\mathrm{Ti}_{0.41} \mathrm{Al}_{0.51} \mathrm{Ta}_{0.08} \mathrm{~N}$ films via hybrid HIPIMS/DC magnetron co-sputtering with synchronized metal-ion irradiation, J. Appl. Phys. 121 (2017) 171902 
[22] G. Greczynski, J. Lu, M. Johansson, J. Jensen, I. Petrov, J.E. Greene, and L. Hultman, Role of $\mathrm{Ti}^{\mathrm{n+}}$ and $\mathrm{Al}^{\mathrm{n}+}$ ion irradiation $(\mathrm{n}=1,2)$ during $\mathrm{Ti}_{1-\mathrm{x}} \mathrm{Al}_{\mathrm{x}} \mathrm{N}$ alloy film growth in a hybrid HIPIMS/magnetron mode, Surf. Coat. Technol. 206 (2012) 4202-4211

[23] G. Greczynski, I. Zhirkov, I. Petrov, J.E. Greene, and J. Rosen, Gas rarefaction effects during high power pulsed magnetron sputtering of groups IVb and VIb transition metals in Ar, J. Vac. Sci. Technol. A 35 (2017) 060601

[24] J. Bohlmark, M. Lattemann, J.T. Gudmundsson, A.P. Ehiasarian, Y.A. Gonzalvo, N. Brenning, U. Helmersson, The ion energy distributions and ion flux composition from a high power impulse magnetron sputtering discharge,

Thin Solid Films 515 (2006) 1522-1526

[25] P. Sigmund, Sputtering of single and multiple component materials, J. Vac. Sci. Technol. A 17 (1979) 396-399

[26] M. W. Thompson, Physical mechanisms of sputtering, Physics Reports 69 (1981) 335-371

[27] J.T. Gudmundsson, N. Brenning, D. Lundin, and U. Helmersson, High power impulse magnetron sputtering discharge, J. Vac. Sci. Technol. A, 30 (2012) 030801

[28] W.D. Westwood, Calculation of deposition rates in diode sputtering systems, J. Vac. Sci. Technol. 15 (1978) 1 

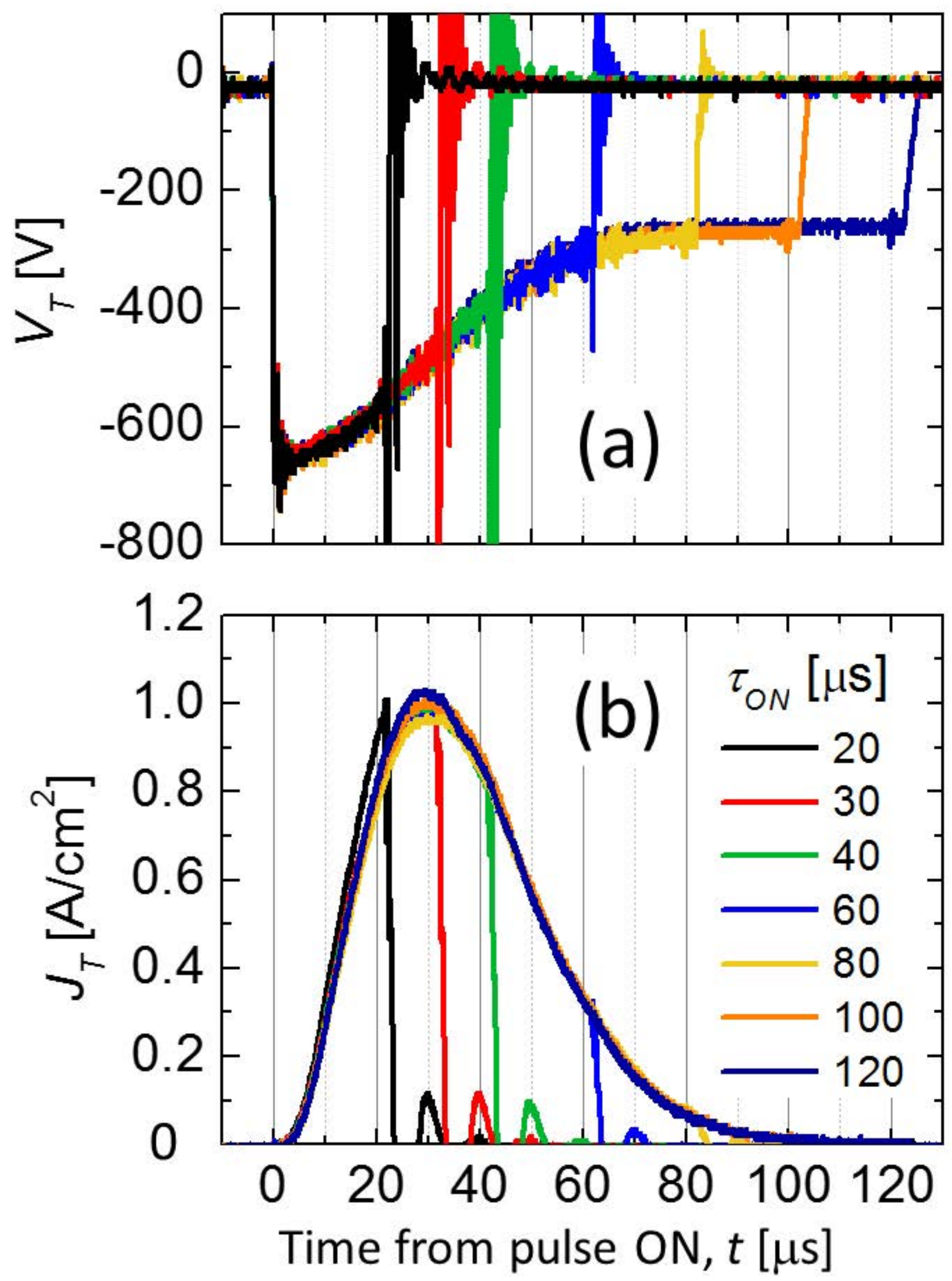

Fig. 1 


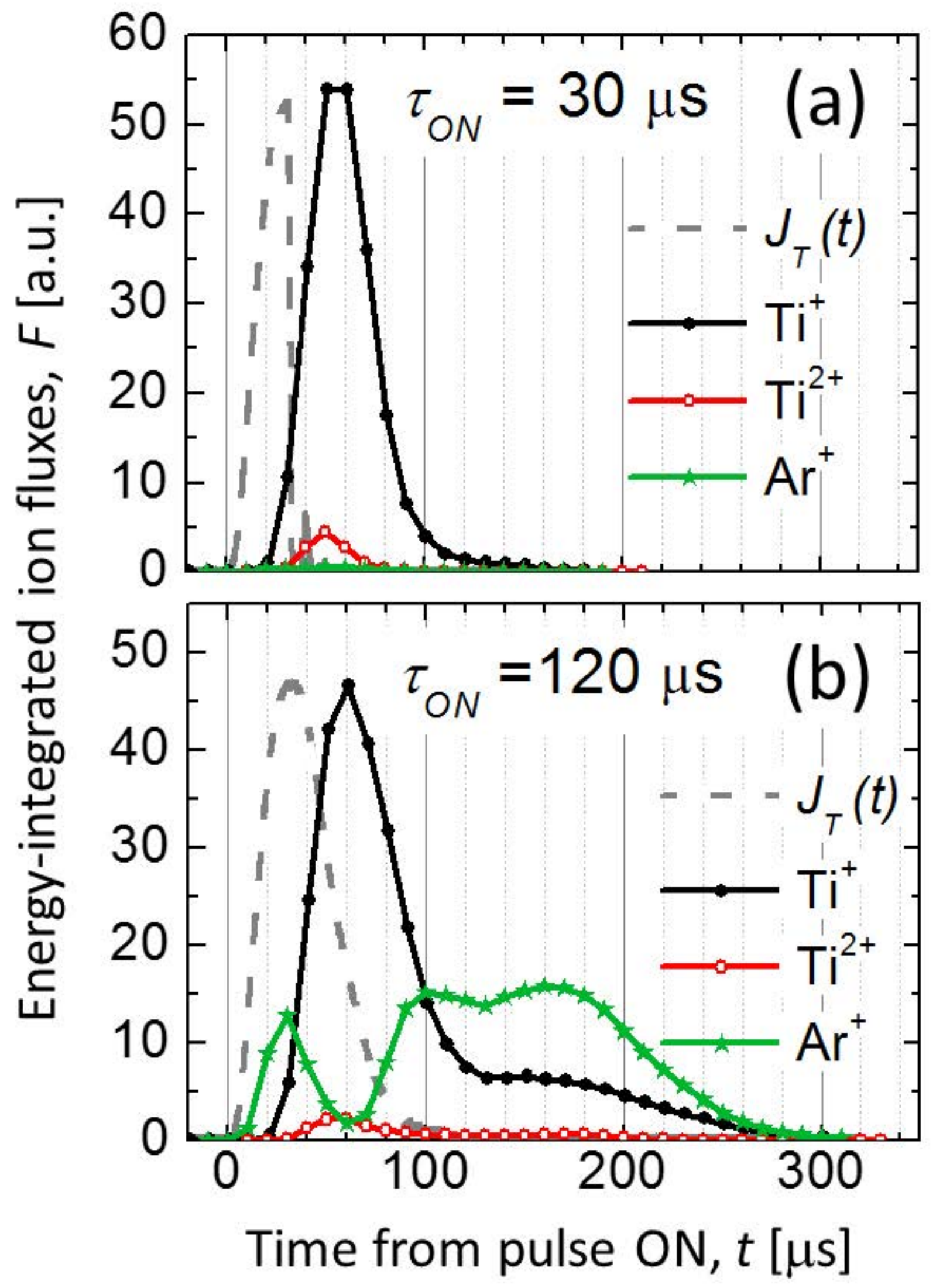

Fig. 2 


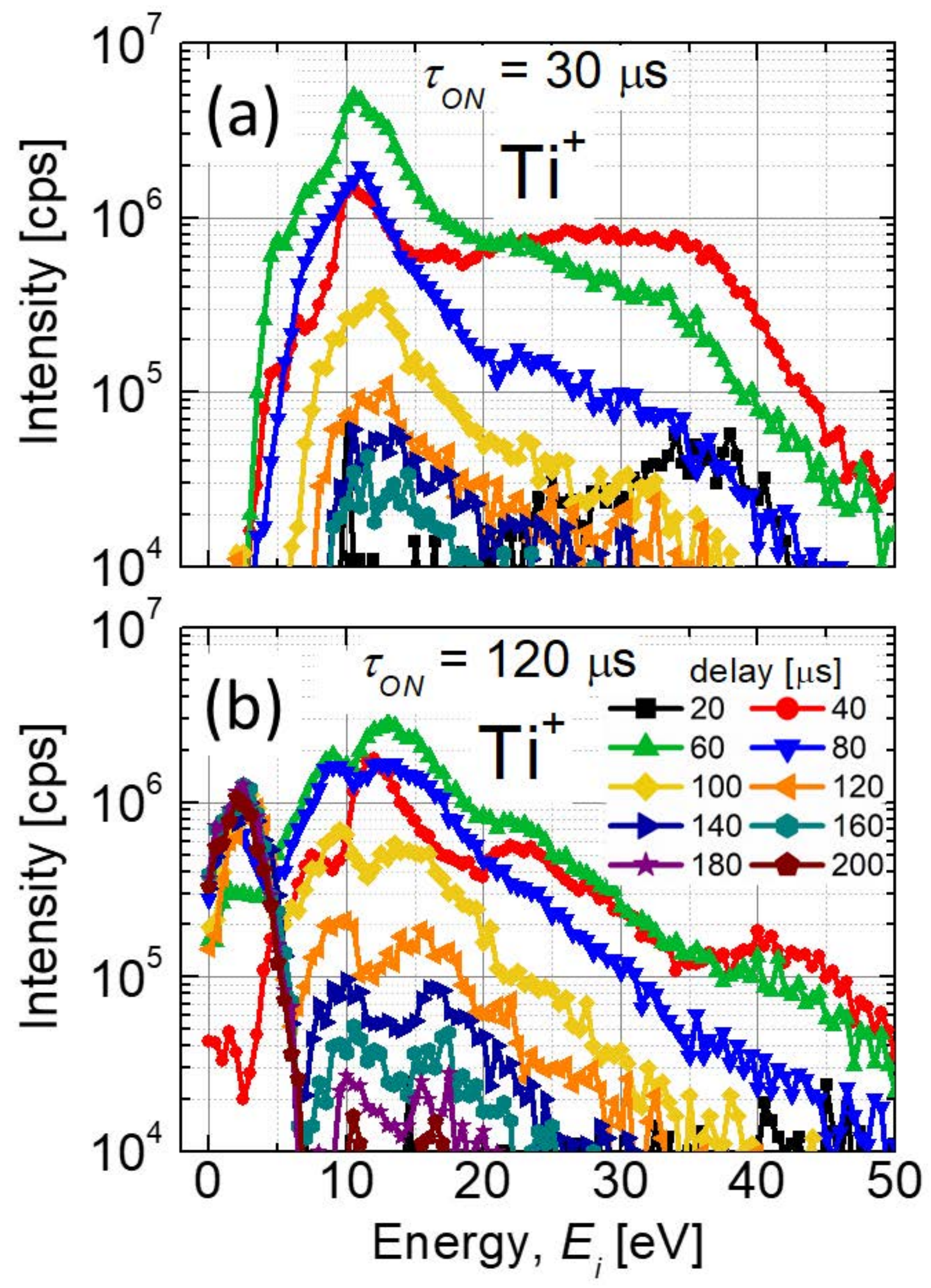

Fig. 3 


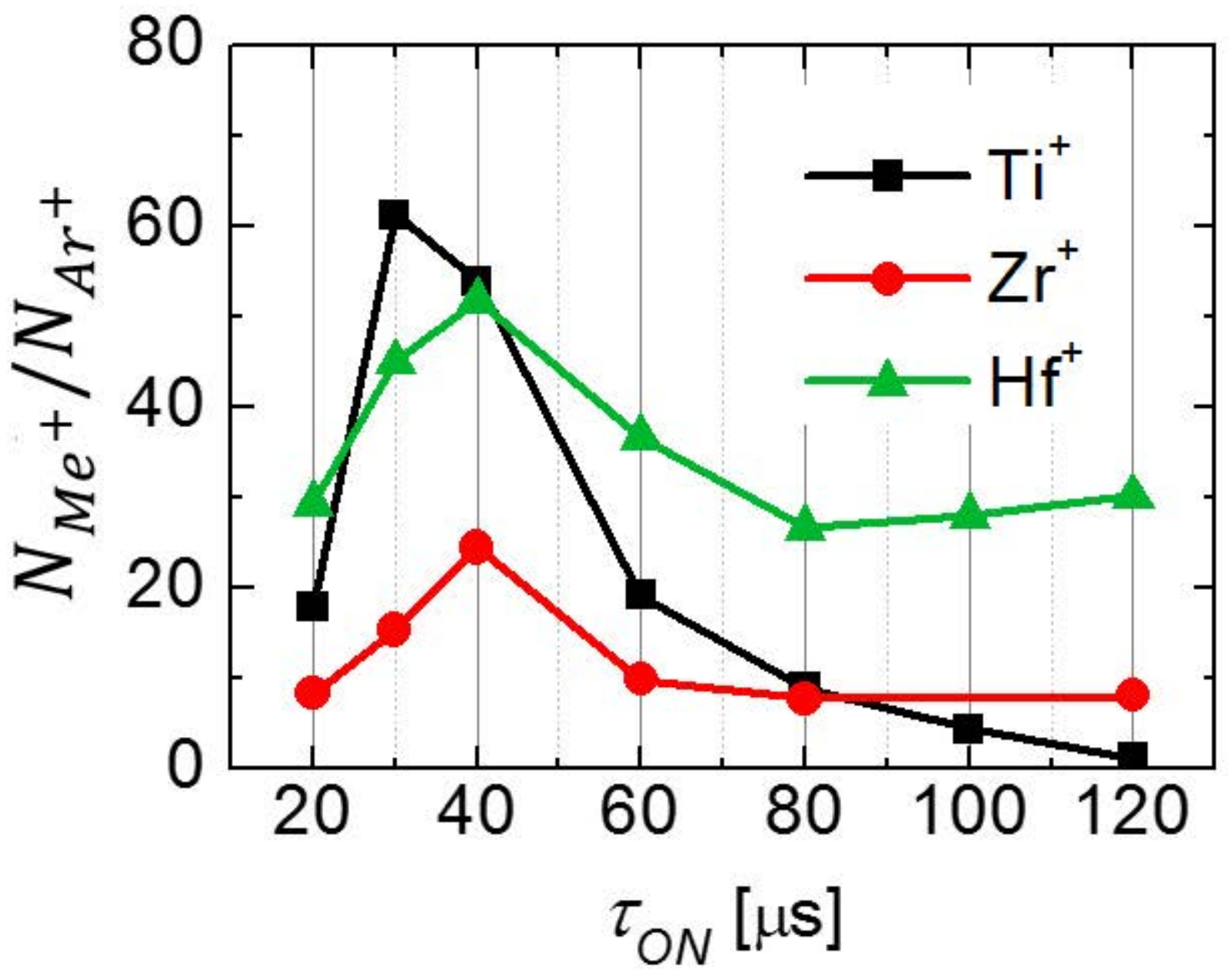

Fig. 4 


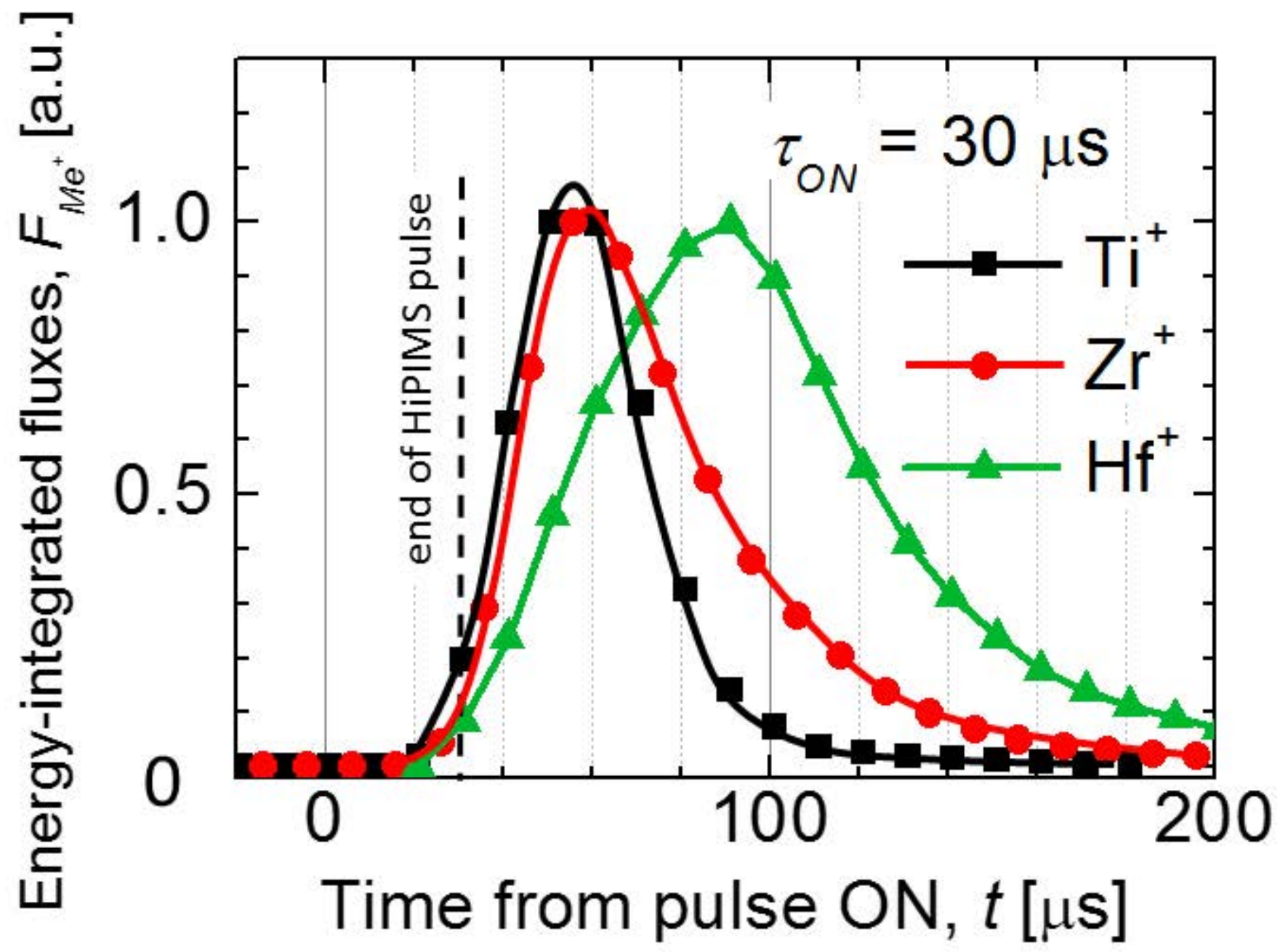

Fig. 5 